\title{
Gouvernance de l'eau potable et dynamiques locales en zone rurale au Bénin
}

\section{Bernard G. Hounmenou}

\section{Q OpenEdition}

Journals

\section{Édition électronique}

URL : http://journals.openedition.org/developpementdurable/1763

DOI : 10.4000/developpementdurable.1763

ISSN : 1772-9971

Éditeur

Association DD\&T

\section{Référence électronique}

Bernard G. Hounmenou, « Gouvernance de l'eau potable et dynamiques locales en zone rurale au Bénin », Développement durable et territoires [En ligne], Dossier 6 | 2006, mis en ligne le 12 mai 2006, consulté le 30 avril 2019. URL : http://journals.openedition.org/developpementdurable/1763 ; DOI 10.4000/developpementdurable.1763

Ce document a été généré automatiquement le 30 avril 2019.

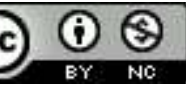

Développement Durable et Territoires est mis à disposition selon les termes de la licence Creative Commons Attribution - Pas d'Utilisation Commerciale 4.0 International. 


\title{
Gouvernance de l'eau potable et dynamiques locales en zone rurale au Bénin
}

\author{
Bernard G. Hounmenou
}

1 L'eau constitue un bien considéré à l'instar de l'air, comme essentiel à la vie humaine. L'accès à cette ressource pose encore d'énormes difficultés dans plusieurs régions du monde. En effet, un milliard quatre cent millions environ d'êtres humains dans le monde, n'avaient toujours pas accès en 2003 à l'eau potable et parmi eux, 450 millions se situaient en Afrique (Gauthier, 2004). Alors qu'environ 85\% de la population urbaine en Afrique a de l'eau potable, $55 \%$ de la population rurale n'y a toujours pas accès (Enterprise Works World Wide, 2003). Il existe un lien direct entre le manque d'accès à l'eau et toutes sortes de maladies dont sont victimes les populations pauvres dans le monde, en particulier dans les pays en voie de développement (Briand et Lemaître, 2004). Les effets néfastes des difficultés d'accès à l'eau potable sur la santé et l'hygiène, constituent aussi un facteur prépondérant dans le cercle vicieux de la pauvreté. Dans de nombreuses régions des pays en développement, la charge d'aller chercher de l'eau revient en effet aux femmes et aux enfants, qui doivent souvent parcourir de longues distances, leur laissant alors moins de temps pour des activités génératrices de revenu comme l'éducation, etc.

2 Le coût élevé du développement des ressources en eau potable, constitue un obstacle majeur à l'amélioration de l'accès à l'eau dans les régions pauvres, en particulier en Afrique rurale. Dans de nombreux cas cependant, les villageois sont prêts à consentir une contribution financière majeure, afin de s'assurer une source locale en eau potable. Les modes de consommation et de production de l'eau subissent en général de nombreuses et importantes transformations, faisant ainsi évoluer de façon manifeste, la place de cette ressource dans le système économique et social. L'augmentation de la demande en eau, débouche sur des problèmes d'allocation de la ressource, ce qui lui donne certaines caractéristiques inhérentes aux biens dits " économiques » : l'eau devient un bien naturel rare. Or, lorsqu'une ressource devient rare, une concurrence émerge du fait des rivalités 
de consommation. Mais la valorisation de la ressource entraînée par cette compétition, ne se manifeste pas uniquement en termes de prix. A cet effet, la directive cadre européenne sur l'eau adoptée en 2000 précise que, «l'eau n'est pas un bien marchand comme les autres, mais un patrimoine qu'il faut protéger, défendre et traiter comme tel »(Calvo-Mendieta, 2004). Derrière le débat sur le caractère marchand ou non de l'eau, se cache la question plus fondamentale de la nature de ce liquide essentiel à la vie et dont l'usage soulève des enjeux importants. Les déterminants de choix des usagers de la ressource, peuvent être différents des règles de l'offre et de la demande (Thaithe, 2001). L'eau présente par ailleurs, en plus de la rivalité à laquelle peut donner lieu sa consommation, la particularité d'être non exclusive dans la mesure où cette consommation n'est pas réservée à un groupe ou à une catégorie de personnes. Ces éléments font de l'eau un bien public impur, en l'occurrence un bien commun ou une ressource commune (Ostrom, 1990).

3 Pendant longtemps, les services liés à la fourniture d'eau potable sont restés en zones urbaines, sous le régime de la propriété publique alors qu'en zones rurales des pays en développement, cette ressource est restée en accès libre, même lorsque des investissements publics sont réalisés dans des aménagements spécifiques, pour la rendre accessible. Avec la mise en œuvre des politiques de libéralisation économique depuis la fin des années 1980, notamment dans les pays du Sud, plusieurs Etats ont fait passer les services d'accès à l'eau potable en zones urbaines, du régime de propriété publique à celui de propriété semi-publique ou privée. Dans les zones rurales des pays concernés, le service d'accès à l'eau potable est enraciné dans nombre de cas, dans un régime de propriété commune. C'est le cas, en particulier, dans plusieurs localités rurales du Bénin, où des équipements collectifs de fourniture d'eau potable sont de plus en plus mis en place, dans le cadre d'une démarche participative venant des populations villageoises, avec l'aide de l'Etat et des organismes d'appui (ONG, bailleurs de fonds,...). La fourniture d'eau potable en zone rurale béninoise fait alors l'objet d'une gouvernance qui s'inscrit dans une perspective de développement durable, rendant les populations rurales propriétaires et gestionnaires des équipements installés. L'étude de cette gouvernance fait l'objet de cet article qui s'appuie sur des expériences conduites dans le domaine de l'eau potable, dans certaines localités des départements de l'Atacora, au nord-ouest du pays, et de l'Atlantique, au sud (Figure 1$)^{1}$. 
Figure 1 : Carte du Bénin

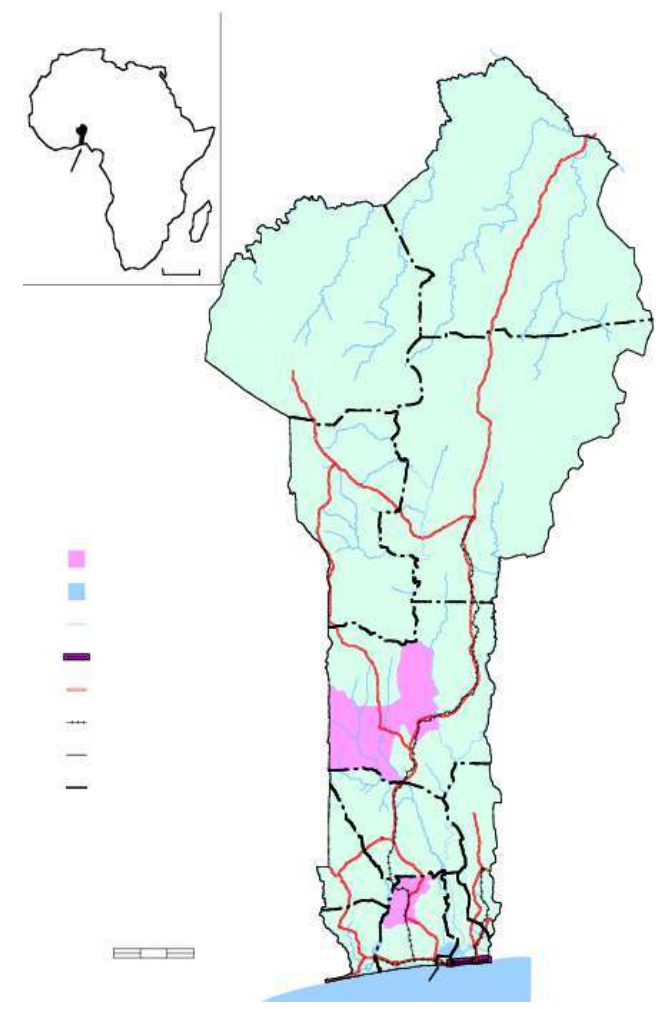

Source : Institut Géographique National (Bénin), 1992

4 Les caractéristiques économiques liées aux ressources en eau les classent dans une catégorie spécifique de biens. Il s'agit de biens communs encore appelés ressources communes. Les biens communs, sont caractérisés par une non-exclusion et une rivalité (Ostrom, 1999). Un bien est non exclusif lorsqu'une fois produit, il est accessible à tous ; aucune minorité n'a l'exclusivité d'accès. La rivalité ou la divisibilité du bien se rapporte au fait que la consommation d'une certaine quantité du bien peut limiter la quantité disponible pour les autres (Samuelson, 1954 ; Cornes et Sandler, 1996). N'importe qui au sein d'un groupe de personnes plus ou moins bien défini, peut utiliser des ressources régies comme biens communs ou s'en débarrasser, selon des règles qui peuvent varier $\mathrm{du}$ «laisser-faire» jusqu'à des règles formelles édictées de façon assez tranchée et effectivement mises en application. Quelques distinctions peuvent être faites au sein des biens communs en fonction des critères tels que l'existence de règles régissant l'utilisation de la ressource et le type de régime de propriété qui lui est applicable.

Suivant l'existence ou non de règles déterminant l'utilisation ou la gestion des ressources collectives, celles-ci peuvent être distinguées en biens communs ouverts et en biens communs réglementés.

Les ressources disponibles suivant ce type de biens peuvent être utilisées à volonté ou gratuitement. Les services de fourniture d'eau à travers les équipements publics dans les villages béninois, peuvent être considérés comme des biens communs ouverts dans la période précédant les années 1990. Avec cette liberté d'accès, la tentation pour les usagers d'agir comme "passager clandestin » est grande comme dans le cas des biens publics. Tout individu pouvait alors user de la ressource publique d'eau, sans contribuer à l'entretien ou à la maintenance des équipements locaux. Lorsque la majorité ou la totalité 
des usagers se comportent de la sorte, il n'y a personne pour œuvrer au fonctionnement durable des équipements de fourniture d'eau. Dans ce contexte, les populations avaient pendant longtemps compté sur le pouvoir central (notamment ses services techniques) pour leur approvisionnement en eau potable. Face aux sollicitations de plus en plus grandes des populations et à cause des difficultés croissantes de l'Etat pour faire face à ses engagements, les usagers de plusieurs localités avaient abandonné leurs équipements hydrauliques tombés en panne, pour se replier sur d'autres sources alternatives d'eau de qualité impropre à la consommation humaine. Cette situation peut être reliée à la célèbre formule inventée par Garett Hardin en 1968, sous l'appellation de la "tragédie des communaux». Cette «tragédie» apparaît comme un paradoxe: "des stratégies individuelles rationnelles conduisent à des résultats collectifs irrationnels »(Ostrom, 1990).

Cette tragédie n'est pas due au caractère commun des ressources, mais plutôt à leur accès libre. Ce que Hardin appelle "communaux» («commons») correspond en fait à des ressources en libre accès, dont les droits de propriété et/ou d'usage n'ont pas été déterminés (Stevenson, 1991 ; Lych, 1999).

Ils sont régis par des règles plus ou moins élaborées, certaines formelles, d'autres, sous forme de conventions sociales régissant l'usage des ressources.

L'accès au service d'eau potable fourni par des équipements collectifs en zones rurales béninoises est actuellement réglementé. Une stratégie nationale définit les conditions de mise en place et de gestion de ces équipements. Cette stratégie préconise l'instauration d'un péage spécifique pour l'accès à l'eau potable fournie à travers les équipements collectifs. Ce péage est en général défini en concertation avec la population des usagers, au prorata du volume d'eau prélevé.

communs en termes d'exclusion et de rivalité fait abstraction des régimes de propriété qui leurs sont applicables. La qualification d'un bien en propriété commune ou en propriété publique a d'importantes incidences sur la perception de ce bien pour le consommateur et sur les stratégies de gestion (Thaithe, 2001). En fonction du sujet détenant le droit de propriété, trois régimes différents peuvent être distingués (Ostrom, 1990 ; Bromley, 1991) :

11 Un agent économique a le contrôle absolu sur la ressource (droit d'usus, de fructus et d' abusus). Les équipements privés aménagés en zones rurales par les individus sur leurs domaines (concessions, exploitations) répondent à ce type de régime. Dans une certaine mesure, la distribution de l'eau au robinet par des particuliers urbains à leur entourage, peut être analysée comme un régime de propriété privée, quand bien même la gestion le l'ensemble du réseau relève du monopole d'une société d'Etat.

12 L'Etat a le contrôle absolu sur la ressource, et définit (directement ou via une agence publique) ses conditions d'usage.

La production et la distribution de l'eau potable en zone urbaine au Bénin répondent à ce régime. En effet, dans les grandes villes béninoises, la fourniture du service d'eau potable est restée jusqu'en 2003, le monopole d'une entreprise publique, l'ancienne Société Béninoise d'Electricité et d'Eau (SBEE). Par le biais de cette société, les investissements sont faits en infrastructures, et l'eau est fournie aux abonnés urbains à travers des branchements privés. Certains abonnés vendent l'eau de façon informelle à leur entourage au robinet, à des prix fixés au volume. Une séparation a été réalisée en 2004 entre les services publics de fourniture d'électricité et ceux relatifs à l'eau potable. Ainsi, 
à partir de cette date, la distribution de l'eau a été spécifiquement déléguée à la Société Nationale des Eaux du Bénin (SONEB).

L'analyse des deux précédents régimes de propriété soulève un problème plus général de la responsabilité de l'allocation des ressources en eau. Sur cette question, deux approches s'appuyant sur des fondements théoriques différents sont généralement utilisées : celles qui prônent le marché comme mécanisme efficace d'allocation des ressources et celles qui défendent l'intervention publique comme moyen d'allocation permettant une minimisation des défaillances du marché. Toutes les deux tiennent leurs sources dans l'assertion que l'absence de régulation conduirait à la surexploitation des ressources par les usagers, qui en abuseraient sans prendre en compte les autres usagers. Face à cette « tragédie » de Hardin et dépassant l'alternative Etat-Marché, la propriété commune apparait comme une troisième solution à ne pas négliger lors de l'analyse de l'allocation des ressources (Ostrom, 1990 ; Stevenson, 1991).

Un ensemble d'individus engagés contractuellement a le contrôle absolu sur la ressource (dont le droit d'exclure les non membres), et peut nommer un arbitre extérieur qui doit faire respecter le contrat. Avec la nouvelle stratégie nationale, les services collectifs de fourniture d'eau potable en zone rurale au Bénin, sont soumis à un régime de propriété commune. Les équipements de fourniture d'eau (forages, réseaux d'adduction villageoise, postes d'eau autonome,...) constituent des propriétés collectives des usagers locaux. L'initiative de l'aménagement de ces équipements doit venir de ces usagers. Dans la mise en place des équipements, ces derniers doivent apporter collectivement leurs participations. Ils sont en outre responsables de l'entretien et de la gestion durable des équipements.

16 Les réflexions sur les ressources en propriété commune (" common-pool resources ») se sont développées à partir des travaux d'Elinor Ostrom (1990), et se basent sur la capacité des institutions communautaires à générer et appliquer des règles d'action collective auto-limitatives. Ce régime suggère que les usagers de la ressource s'engagent par contrat, à mettre en œuvre une stratégie coopérative définie par eux, confiant à un acteur (arbitre) externe, la charge de faire respecter le contrat.

17 Au-delà d'Ostrom $(1990,1999)$, de nombreux auteurs s'opposant aux arguments d'Hardin et de ses défenseurs sur l'impossibilité d'une action collective (Berkes et al, 1989; Stevenson, 1991) ont indiqué qu'une ressource peut faire l'objet d'une gestion durable par la communauté. Ils ont aussi présenté les fondements d'une approche institutionnelle qui met l'accent sur les mécanismes de régulation formels ou informels, qui gouvernent la viabilité des systèmes naturels "anthropisés ». Calvo-Mendieta (2004) mentionne à cet effet qu'il existerait des circonstances dans lesquelles, les régimes de propriété commune sont pérennes, et peut-être même souvent plus efficaces que les régimes de la propriété individuelle. Analysant les conclusions d'Hardin, les défenseurs du régime de propriété commune trouvent qu'il serait plus pertinent de parler de tragédie du libre accès, afin de préciser que c'est l'absence complète de droits établis, qui peut avoir comme conséquence la destruction des ressources et non pas une situation où la propriété des ressources est commune.

18 Ainsi, si dans le passé (jusqu'à la fin des années 1980), les équipements de fourniture d'eau potable étaient abandonnés faute d'entretien par les populations d'usagers en zones rurales, ce n'est pas leur propriété collective qui en était la cause. C'est le fait plutôt de l'insuffisance de responsabilisation des usagers dans la gestion de ces équipements à 
travers la définition, l'adoption et le respect de règles régissant l'utilisation de la ressource en eau.

La gouvernance renvoie à l'intervention combinée d'acteurs variés sur un territoire et à la capacité de ce système d'acteurs de produire des politiques publiques cohérentes. Elle fait appel à la fois à une coordination des actions entreprises par les acteurs, ce qui nécessite donc un processus de synchronisation tel que la planification, mais aussi une cohérence qui sous-entend la reconnaissance des finalités communes à atteindre (Bertacchini, 2002). La gouvernance renvoie non seulement à des actions collectives, mais aussi à une responsabilisation des différents acteurs et aux relations de partenariat entre ceux-ci, dans le cadre de la promotion économique et sociale du territoire. Ces actions impliquent un ensemble d'institutions dont les collectivités locales, les services déconcentrés de l'Etat, les institutions de la société civile ou des milieux professionnels, le secteur privé, ... La gouvernance peut impliquer la mobilisation des acteurs dans divers types d'actions relatives à la vie de la population d'un territoire. Elle peut aussi être sectorielle et se rapporter à des actions liées à un secteur spécifique. C'est le cas par exemple du secteur des services liés à l'approvisionnement en eau potable.

Jusqu'à la fin des années 1980, des ouvrages d'approvisionnement en eau potable ont été construits en milieu rural béninois, avec la seule initiative des pouvoirs publics. Dans le cadre de l'implantation de ces ouvrages, les communautés bénéficiaires n'étaient pas suffisamment associées, ni suffisamment préparées à leur entretien. Cette situation se traduisait par une insuffisance d'appropriation des populations dans la gestion des équipements en cause avec comme conséquence, l'abandon des ouvrages en cas de panne et le recours aux sources d'eau non potable. Pour améliorer la situation, le Bénin a adopté en 1992 une stratégie nationale d'alimentation en eau potable. Cette stratégie vise à impliquer les populations du monde rural dans tout le processus d'appropriation de l'alimentation en eau potable. Les principes fondamentaux de cette stratégie sont en particulier, la décentralisation du processus de prise de décision, ainsi que la participation des communautés à l'investissement et à la gestion des points d'eau.

Dans le cadre de la stratégie, la demande des communautés est le point de départ du processus de réalisation des ouvrages. Les communautés doivent pouvoir faire ellesmêmes le choix du type d'ouvrage d'eau à réaliser chez elles. Cette demande est en général formulée avec la coordination et la validation des chefs de villages et d'autres responsables villageois (sages, responsables d'organisations). Pour se donner la capacité d'assurer l'entretien, la maintenance et le renouvellement des ouvrages, l'eau fournie est mise en vente par les communautés et les recettes sont épargnées.

La concrétisation de la volonté d'améliorer les systèmes d'alimentation en eau potable se traduit par l'adhésion de la communauté bénéficiaire au cofinancement des ouvrages, à la mise en place d'une structure de gestion des équipements réalisés. Cette structure est aussi chargée d'œuvrer à l'entretien et à la maintenance des ouvrages, ainsi qu'à la promotion de l'hygiène et de l'assainissement autour de ceux-ci. La mobilisation des contributions financières des populations dans le cadre de la mise en place des équipements de fourniture d'eau potable, passe en général par des cotisations effectuées par les ménages, des souscriptions volontaires, des dons effectués par certains membres d'associations locales de développement, etc. En zones de production cotonnière (villages au centre et au nord du pays), les groupements villageois jouent un important rôle dans cette mobilisation. Ces groupements fournissent souvent le cofinancement local sur la base des prélèvements qu'ils opèrent sur les ristournes cotonnières. 

lors de la mise en place d'équipements hydrauliques, celles-ci continuent à bénéficier du concours financier de l'Etat et de certains bailleurs. C'est le cas par exemple, avec le projet d'hydraulique villageoise dont les actions ont été réalisées dans les années 1980, avec le soutien de l'Etat béninois et de la coopération japonaise. Les actions du Projet d'Appui au Développement de l'Eau et de l'Assainissement en milieu Rural (PADEAR) ont aussi été mises en œuvre à partir des années 1990, dans le cadre d'un partenariat entre l'Etat béninois et les bailleurs de fonds (Banque Mondiale, Coopération danoise, Coopération allemande, Coopération belge, ..). Dans la conduite de ces actions, une assistance technique est apportée aux populations rurales par les services déconcentrés de l'Etat. Cette assistance prend en compte la formation des artisans réparateurs sur les territoires villageois. L'objectif est de former et d'installer dans chaque territoire communal, un certain nombre d'artisans réparateurs afin que ceux-ci assurent, dans une logique professionnelle, des prestations de service aux populations villageoises dans le domaine de l'entretien technique de leurs équipements hydrauliques. Pour favoriser une maintenance régulière des équipements hydrauliques, les fournisseurs privés sont encouragés à ouvrir des dépôts de pièces de rechange, dans la plupart des territoires des actuelles communes rurales. Outre les soutiens techniques et financiers, les populations villageoises bénéficient ces dernières années des appuis organisationnels des ONG. Cellesci se chargent en général de sensibiliser, de former et de mobiliser la participation des villageois dans la mise en place et la gestion des équipements hydrauliques. Certaines de ces ONG interviennent à titre d'intermédiaires dans la mise en œuvre de programmes hydrauliques (cas du PADEAR). collectivité territoriale décentralisée, la responsabilité de réaliser les infrastructures au profit des populations de son territoire (article 83 de la loi 97-029). Ainsi à partir de 2004, dans le cadre de l'élaboration des Plans de Développement dans les communes, de nombreux conseils communaux ont (à travers les consultations des populations et de divers acteurs sur leurs territoires) programmé la réalisation d'ouvrages hydrauliques, en vue d'améliorer les services publics liés à la fourniture d'eau potable. Cependant, sur certains territoires communaux, les collectivités locales intervenaient déjà avant l'avènement de l'actuelle décentralisation, dans l'amélioration des conditions d'accès à l'eau potable des populations. C'est le cas par exemple dans quelques communes du département des Collines (Savalou, Dassa-Zoumè), avec les actions menées dans le cadre des projets de territoire. Dans ces communes, la mise en place des équipements de fourniture d'eau potable a été proposée par les populations villageoises. L'opportunité et la faisabilité de la mise en œuvre de ces priorités ont été analysées par des groupes thématiques mettant en concertation les représentants villageois, les autorités communales, les représentants des services techniques de l'Etat, les techniciens du secteur privé et les animateurs d'autres structures intervenant dans le secteur (ONG). Les actions découlant des projets de territoire sont mises en œuvre avec un cofinancement de la commune, dans le cadre d'une maîtrise d'ouvrage assurée par celle-ci. L'analyse du système de gouvernance de l'eau potable en zone rurale béninoise fait ainsi apparaître trois niveaux de régulation: 1) Le niveau national avec l'intervention de l'Etat notamment dans la planification nationale, la réglementation du secteur de l'eau et l'appui technique, financier et organisationnel aux communautés villageoises. 2) Le niveau communal: la collectivité locale assume l'une des compétences qu'elle partage 
avec l'Etat dans le cadre de la décentralisation. A travers son plan de développement et son budget, la commune met en œuvre les priorités de ses populations en matière d'approvisionnement en eau potable. 3) Le niveau de la communauté villageoise : c'est à ce niveau que les populations bénéficiaires apportent leur participation (cofinancement, apport de main d'œuvre non qualifiée et de matériaux locaux), à la mise en place des équipements. C'est aussi à ce niveau que les usagers sont impliqués dans une dynamique de gestion pérenne des équipements dans leurs localités.

L'eau distribuée par des équipements collectifs aménagés dans les villages, est soumise à un régime de propriété collective. Même si un cadre de référence du dispositif institutionnel relatif à la gestion des équipements hydrauliques est défini et imposé par l'Etat, les membres de la communauté villageoise s'engagent contractuellement entre eux, pour contrôler et gérer en commun leurs ressources en eau. C'est notamment dans cet esprit qu'ils mettent en place une structure communautaire de gestion d'équipements (association d'usagers, comité de gestion). Le respect de ce contrat se fait non seulement à travers le recours à la compétence d'un exploitant rémunéré, mais aussi grâce aux services hydrauliques de l'Etat. Tout en assurant une assistance technique à la communauté villageoise, ces derniers veillent aussi au respect des clauses du contrat, et à la conformité des actions menées avec les textes réglementaires dans le domaine de la gestion communautaire des infrastructures hydrauliques.

L'implication active des populations bénéficiaires dans la gestion des équipements hydrauliques, constitue actuellement une sérieuse option, visant à favoriser leur accès durable à l'eau potable. Cette implication passe, dans une large mesure, par l'émergence au sein de ces populations, de structures de gestion chargées de l'entretien et de la maintenance des équipements. Ces structures constituent aussi des instances représentant les populations dans toutes les affaires relatives au service d'accès à l'eau potable. Elles ont par ailleurs pour tâches de mobiliser la participation financière des populations à la construction des ouvrages, la fixation du prix, le choix des vendeurs d'eau, l'entretien et la maintenance des équipements, etc. Pour un ouvrage simple comme un puits moderne ou un forage équipé de pompe à motricité humaine, aménagés pour desservir des populations de petite taille (un peu plus de 250 habitants), la structure de gestion reste légère. Elle prend la forme de comité de gestion, structure de petite taille, constituée de personnes désignées par la population des usagers du point d'eau. Pour un ouvrage complexe comme une Adduction d'Eau Villageoise (AEV) ou un Poste d'Eau Autonome (PEA), qui sont aménagés pour une population beaucoup plus nombreuse (1000 à 1500 personnes pour un PEA et plus de 1500 personnes pour une AEV), la structure de gestion prend la forme d'une Association des Usagers d'Eau (AUE). Dans une localité villageoise où il $\mathrm{y}$ a à la fois une AEV ou un PEA et en plus, des puits modernes ou des forages de pompe à motricité humaine, la gestion de l'ensemble des ouvrages est assurée par une AUE.

Dans certaines localités rurales de plusieurs pays de l'Afrique sub-saharienne, le modèle d'approvisionnement en eau potable, représenté par les petits ouvrages comme les puits et forages, tend à reculer au profit des réseaux intervillageois d'ouvrages complexes. Ces derniers permettent en effet de compenser le manque d'eau localisé. Les villageois ne possédant pas de forage sont approvisionnés à partir de villages voisins qui en possèdent. Il se met ainsi progressivement en place le passage d'une alimentation en eau ponctuelle (par puits ou forage) à une alimentation en eau par réseau à travers laquelle les villages sont davantage connectés les uns aux autres (Briand et Lemaitre, 2004). 

indicatif, l'eau est livrée par l'AUE de Houègbo (commune de Toffo) au niveau des bornes fontaines à $300 \mathrm{FCFA}^{2}$ le mètre cube au cours de l'année 2004. Elle est vendue en détail aux ménages, au niveau de ces points d'eau publics, au prix de 15 FCFA la bassine de 35 litres. Le prix de cession pratiqué par l'AUE aux abonnés privés est de 290 FCFA le mètre cube. Toutefois, les petites maintenances telles que celles liées au remplacement d'un robinet, sont à la charge de l'abonné privé. populations bénéficiaires organisées en Association des Usagers d'Eau (AUE). Cette association volontaire regroupe l'ensemble des villageois usagers d'eau, résidant sur le territoire (un ou plusieurs villages), desservi par un système d'adduction (AEV ou PEA). Cette association a plusieurs missions, notamment: 1) Promouvoir et préparer la réalisation d'une adduction d'eau villageoise, en particulier mobiliser la participation de la communauté à l'investissement initial ; 2) assurer le service public de distribution d'eau potable; 3) représenter les usagers pour l'ensemble des décisions relatives au service d'eau potable de la communauté ; 4) déléguer la gestion quotidienne du système à un exploitant et passer un contrat de maintenance avec une entreprise agréée; 5) et gérer les équipements pour assurer à travers la vente de l'eau potable au volume, la pérennité et le renouvellement des équipements. fontaine concernée. Le comité de point d'eau est chargé d'assurer l'entretien de la borne fontaine et l'assainissement de son site d'implantation. Il assure le recrutement et le suivi d'un fontainier, pour la vente de l'eau au niveau de la borne fontaine. Le comité de point d'eau dispose d'un fonds, qui rémunère le fontainier au prorata de la quantité d'eau vendue. L'ensemble des représentants de tous les points d'eau publics du système constitue l'assemblée générale de l'AUE. Cette assemblée élit un comité directeur pour la gestion du système, la représentation des femmes et les critères liés au développement local, étant particulièrement valorisés dans la composition de ce comité.

Dans le cas des postes d'eau autonomes où il existe une seule fontaine, une seule structure est mise en place, jouant à la fois le rôle de comité de point d'eau et de comité directeur. Ses membres sont désignés en assemblée, par un collège électoral constitué de personnes déléguées par la population de chaque quartier du village. L'assemblée générale de l'AUE choisit le mode d'exploitation, fixe le prix de l'eau et approuve le budget prévisionnel, sur proposition du comité directeur. Elle décide des propositions de renouvellement et d'extension des équipements.

La valorisation de l'eau par le prix est un enjeu fondamental pour la pérennité de sa fourniture à la population. Elle permet non seulement à celle-ci, de supporter les charges d'exploitation et de maintenance, mais aussi de réaliser des investissements nécessaires au développement des réseaux (Briand et Lemaitre, 2004). L'un des inconvénients à ce mode de fonctionnement pour certaines populations rurales béninoises non culturellement habituées à l'achat de l'eau, est le risque de priver par moment les ménages sans ressources financières, de l'accès à ce bien si essentiel dans la satisfaction de leurs besoins vitaux. 

comité directeur utilise le service d'un exploitant salarié. Celui-ci est sélectionné sur la base d'un test de recrutement, et formé par la Direction de l'Hydraulique, avant d'être mis à la disposition du comité directeur qui le rémunère. L'exploitant organise et assure les tâches techniques d'exploitation. Il s'occupe de la production, du traitement et de la distribution de l'eau. Il contrôle les fontainiers, relève les compteurs et encaisse les recettes de vente d'eau pour le compte du comité directeur. Dans certains cas, l'exploitation de l'ensemble du système est confiée par le comité directeur, à un opérateur indépendant (exploitant-fermier), suivant un dispositif de contrat d'affermage. Dans ce cas, l'exploitant-fermier paie périodiquement une redevance à l'AUE. Il recrute et rémunère les fontainiers. Il assure aussi l'entretien et les tâches techniques d'exploitation du système. bénéficier des frais de mission et des primes de rendement à la fin de chaque exercice. De même, les membres des comités de points d'eau sont des bénévoles, mais ils reçoivent une prime annuelle que leurs octroient les assemblées des usagers des bornes fontaines considérées, à partir des excédents de leurs caisses. La pratique généralisée du bénévolat pour les membres des comités directeurs des AUE, n'est pas toujours sans incidence sur le fonctionnement du dispositif institutionnel. En effet, les usagers n'ont pas toujours les moyens de contrôler la gestion de ces comités. Il arrive parfois que certains membres de comités directeurs s'entendent avec les exploitants pour détourner les fonds de leurs AUE à des fins personnelles. Cette situation donne lieu à des conflits au sein de certaines associations d'usagers.

L'association des usagers de points d'eau est soumise à la tutelle du Ministère chargé de l'hydraulique. Dans le cadre de cette tutelle, le Ministère assure le contrôle de la gestion financière et du fonctionnement de l'AUE. L'Etat, à travers ce ministère, assure la maîtrise d'ouvrage de l'équipement d'eau potable. Avec le temps, cette maîtrise d'ouvrage devra être transférée aux communes. Après réalisation, le maître d'ouvrage cède, par signature d'une convention, la propriété et l'exploitation des équipements d'exhaure et des ouvrages de distribution à l'AUE. Les ouvrages de captage de l'eau restent la propriété du maître d'ouvrage et leur renouvellement lui incombe.

Dans le cadre de la maintenance des équipements, la Direction de l'Hydraulique forme dans les villages des artisans réparateurs pour assurer des prestations de service aux AUE lors de petites pannes de système. Pour les grosses réparations, le comité directeur est appelé à introduire un dossier de demande d'intervention technique à la Direction de l'Hydraulique. Celle-ci envoie alors un technicien pour l'établissement d'un diagnostic et l'évaluation financière de l'intervention à réaliser. Le comité directeur est ensuite appelé à opérer le décaissement de fonds pour la remise en service de l'équipement. Outre l'entretien et la maintenance des équipements, beaucoup d'AUE arrivent à investir dans l'extension de leurs réseaux ou dans le cofinancement de diverses infrastructures sociocommunautaires dans leurs localités. Quelques réalisations des AUE sont présentées, à titre indicatif, dans le tableau suivant : 
Tableau 1 : Exemple de réalisations des AUE dans leurs localités.

\begin{tabular}{|c|c|c|c|}
\hline $\begin{array}{l}\text { Localité/Arrondis- } \\
\text { sement de l'AUE }\end{array}$ & Commune & $\begin{array}{l}\text { Types d'actions } \\
\text { financées }\end{array}$ & $\begin{array}{l}\text { Année de } \\
\text { réalisation }\end{array}$ \\
\hline \multirow[t]{2}{*}{ Houègbo/Colli } & \multirow[t]{2}{*}{ Toffo } & $\begin{array}{l}\text { Construction d'un module } \\
\text { de classes en milieu } \\
\text { scolaire }\end{array}$ & 2000 \\
\hline & & $\begin{array}{l}\text { Construction d'une } \\
\text { bibliothèque scolaire }\end{array}$ & 2001 \\
\hline Sèhouè & Toffo & $\begin{array}{l}\text { Travaux de raccordement } \\
\text { d'un village (Gbédji) au } \\
\text { réseau d'adduction d'eau }\end{array}$ & 2002 \\
\hline \multirow{3}{*}{ Ahouannonzou } & \multirow{3}{*}{ Allada } & $\begin{array}{l}\text { Apport d'un cofinancement } \\
\text { de } 2 \text { millions de FCFA au } \\
\text { projet d'électrification de } \\
\text { l'arrondissement }\end{array}$ & 2002 \\
\hline & & $\begin{array}{l}\text { Apport d'un cofinancement } \\
\text { de } 3 \text { millions de FCFA au } \\
\text { projet d'extension d'un } \\
\text { poste d'eau autonome en } \\
\text { mini-réseau d'adduction } \\
\text { villageoise }\end{array}$ & 2003 \\
\hline & & $\begin{array}{l}\text { Réhabilitation du siège de } \\
\text { l'arrondissement }\end{array}$ & 2003 \\
\hline Lahotan & Savalou & $\begin{array}{c}\text { Apport d'un cofinancement } \\
\text { d'un million de FCFA aux } \\
\text { travaux de réfection de } \\
\text { salles de classe en milieu } \\
\text { scolaire }\end{array}$ & 2002 \\
\hline
\end{tabular}

Sources : Travaux de thèse Hounmenou (à paraître)

en début d'année, au vote de son assemblée générale. Celle-ci procède, en fin d'exercice, à l'analyse du bilan des activités menées. Le processus de bilan part en début d'année, des points d'eau. En effet, à cette période, chaque comité de point d'eau présente en assemblée générale à ses usagers, le bilan annuel des activités de gestion d'eau au niveau de sa borne fontaine. A la suite des bilans des comités de point d'eau, le comité directeur présente à l'assemblée générale de l'AUE, le bilan global des activités de gestion de l'eau, sur tout le territoire de l'association.

L'investissement des AUE dans le financement des initiatives en développement dans leurs localités se fait avec l'avis de la Direction départementale de l'Hydraulique, qui assure le suivi et le contrôle de leur gestion. En cas d'avis favorable, l'AUE peut engager des fonds dans l'action concernée. Ceci amène un responsable de l'AUE de Houègbo (commune de Toffo) à affirmer: "En ce qui concerne le pouvoir de décisions relatives à la gestion de l'eau potable en zones rurales, l'Etat béninois n'a pas tout cédé aux populations. Il continue à garder sur lui certaines prérogatives » (Hounmenou, à paraître). Il ressort ainsi que l'autonomie dont bénéficient les associations d'usagers pour la production et la gestion des services liés à l'eau potable dans leurs localités n'est que partielle. Il s'agirait beaucoup plus pour les AUE, d'un exercice de compétences déléguées par l'Etat.

Le mode de fonctionnement des AUE semble les rapprocher des entreprises sociales définies par le réseau EMES $^{3}$. Ce réseau souligne en effet, dans la définition des entreprises sociales, des facteurs constitutifs de la dynamique entreprenariale tels, l'activité continue de production de biens et de services, le degré d'autonomie, une prise de risque économique ou un niveau minimum de travail rémunéré (OCDE, 1999). Certains 
indicateurs ont permis au réseau de parvenir à une définition plus approfondie des entreprises sociales, notamment: l'initiative émanant d'un groupe de citoyens; la répartition du pouvoir non fondé sur la propriété du capital ; la dynamique participative impliquant les destinataires de l'activité ; la distribution limitée des profits éventuels et la finalité explicite de service à la collectivité.

Il s'agit des entreprises viables, c'est-à-dire capables d'équilibrer leur budget grâce à l'alliance de ressources marchandes, non marchandes, non monétaires (recours au bénévolat) et de dons privés, par le biais des meilleures combinaisons possibles.

La gestion des services de fourniture d'eau potable par les AUE fait ressortir, une dynamique entreprenariale avec le recours au travail rémunéré des exploitants, l'utilisation des ressources marchandes (recettes issues de la vente de l'eau), les apports bénévoles des membres (ressources non monétaires). La mise en place des équipements fait aussi souvent intervenir des ressources non marchandes telles, les subventions de l'Etat et de bailleurs internationaux et des cotisations financières des membres de la communauté villageoise. La combinaison de ces différentes ressources permet aux AUE d'équilibrer leurs budgets et de prendre des initiatives, notamment dans le sens de l'extension des équipements et du cofinancement de programmes de développement des communautés. La référence aux initiatives émanant des usagers et l'usage de la démocratie participative, notamment dans le choix des leaders (membres du comité directeur), l'analyse des bilans d'activités et le vote du budget, constituent d'autres éléments qui semblent assimiler les AUE à des entreprises sociales de l'eau aux Bénin.

Dans certaines communes, les AUE sont regroupées au sein d'une union communale. C'est le cas par exemple de la commune de Toffo. Dans le département de l'Atlantique, ces unions communales se sont regroupées dans une fédération départementale. Les activités conduites dans cette fédération sont, pour l'instant, essentiellement liées aux échanges et à la circulation des informations. L'objectif poursuivi par les AUE à travers ces regroupements, est par exemple de défendre leurs intérêts communs auprès des services publics de l'hydraulique qui jusque là, essaient de leur imposer le recours à certains techniciens de maintenance agréés, qui ne leur donnent pas toujours satisfaction. Dans les faits, les actions collectives menées par les AUE dans le cadre de ces regroupements restent très limitées. Cette situation peut être expliquée par la pratique assez répandue qui amène les individus ou leurs organisations à se regrouper pour rechercher des objectifs à poursuivre ensemble. Cette façon de faire va à l'encontre de la logique développée par certaines théories sur la conduite d'actions collectives. Olson (1965) indique que les organisations existent pour permettre d'atteindre les buts qu'un groupe d'hommes a en commun. Ainsi, une organisation viable présuppose normalement un intérêt que partagent ses membres. Dans les localités ne disposant que de simples ouvrages, la mobilisation des populations en vue d'obtenir un service collectif durable de fourniture d'eau potable est aussi notable.

Dans le cas des ouvrages simples tels que les pompes à motricité humaine, la structure de gestion est un comité élu en assemblée générale. Cette dernière est constituée de tous les membres majeurs des ménages usagers du point d'eau. Les membres du comité de gestion sont aussi des bénévoles. Ils sont souvent composés d'un président, d'un secrétaire et d'un trésorier. Dans la plupart des localités villageoises, ce comité comporte également un responsable technique (le Responsable Villageois de Pompe, RVP) et une chargée d'hygiène (en général, une femme). C'est le cas par exemple dans des localités de Natitingou, Tanguiéta, Cobly, Matéri, Boukoumbé, Toukountouna, Wassa-Péhunco, 
Kouandé et Kérou. La responsable d'hygiène a pour rôle d'organiser la propreté autour du point d'eau. Elle organise les femmes usagers du point d'eau, pour le nettoyage dans son environnement immédiat. Elle peut négocier l'appui des hommes pour l'accomplissement de tâches plus exigeantes en main d'œuvre, telles que le débroussaillage. Elle reçoit des formations sur les conditions d'hygiène relatives à la consommation d'eau potable et au maintien d'un environnement sain autour du point d'eau. Elle organise en retour, à travers des séances d'Information-Education-Communication (IEC), la formation des femmes usagers du point d'eau, sur les techniques qu'elle a assimilées. Le RVP s'occupe de l'entretien de l'équipement au niveau du village. Il est formé par un artisan réparateur, sous la supervision du fournisseur de la pompe. Le RVP doit pouvoir détecter les pannes et remplacer les pièces d'usure de la partie aérienne de la pompe. Lorsqu'une panne intervient au niveau de la partie souterraine, il fait appel à l'artisan réparateur, habilité à intervenir sur les deux parties de l'équipement.

L'artisan réparateur fait des visites périodiques pour le suivi de la maintenance de la pompe. Lors des visites, il vérifie le fonctionnement de la pompe. Il conseille le comité de gestion sur le remplacement des pièces et sur les travaux à effectuer sur la pompe. Un artisan réparateur a à sa charge un certain nombre de pompes à suivre (en moyenne, 25 dans l'Atacora). Ainsi, le nombre total de pompes présentes sur le territoire communal détermine le nombre d'artisans réparateurs à promouvoir. L'artisan réparateur doit réaliser tous les quatre mois, une visite périodique à chacune des pompes placées sous son contrôle technique. A chaque visite, il perçoit auprès du comité de gestion, ses frais de déplacement et des frais de prestation déterminés sur la base d'un forfait (qui varie entre 1000 et 1500 FCFA). Lorsque, au cours de ses visites, il détecte une panne ou une difficulté technique au niveau de la pompe, il réunit le comité de gestion pour information. Il reçoit l'avis favorable du comité avant toute intervention technique sur la pompe. Il peut alors, dans ces conditions, démonter la pompe et la réparer sur financement du comité de gestion. L'achat des pièces de rechange est en principe confié au responsable villageois de la pompe. Lorsque la population des usagers fait confiance à l'artisan réparateur, elle peut lui confier le soin d'acheter les pièces. Les informations sur les prix des différentes pièces de rechange, sont généralement mises à la disposition des membres des comités de gestion, par les services techniques et les animateurs des autres institutions (ONG) intervenant dans le secteur.

L'entretien et la maintenance réguliers des équipements, nécessitent la mobilisation de moyens financiers par la population des usagers. Dans cette optique, celle-ci est obligée d'ouvrir un compte d'épargne collectif, avant l'installation ou la remise en service du point d'eau. Dans le département de l'Atacora, la participation financière villageoise s'élève en général à 180000 FCFA pour une réhabilitation d'ancien forage et à 200000 FCFA pour la création d'un nouveau forage équipé de pompes manuelles. Cette somme sert entre autres, à l'aménagement de la superstructure en maçonnerie, du canal d'évacuation ou du déversoir. La population d'usagers procède aussi, dans certains cas, à l'aménagement autour du point d'eau et d'un enclos pour le protéger contre des animaux en divagation.

Lors de la création de nouveaux forages (ou de la réhabilitation d'anciens points d'eau), la participation financière villageoise est mobilisée de différentes manières. Dans plusieurs villages, des cotisations sont effectuées par tous les habitants majeurs (hommes et femmes) des ménages usagers du point d'eau. Dans ce cadre, certaines populations arrivent à négocier le soutien financier (subventions) des organisations villageoises telles 
que, les associations de développement, constituées de ressortissants villageois résidant en majorité dans les agglomérations urbaines. Dans les villages de producteurs cotonniers (cas de Kérou et de Ouassa-Péhounco par exemple), ce sont surtout les groupements villageois qui mobilisent le cofinancement, à travers des prélèvements opérés sur les ristournes cotonnières de leurs membres.

Pour l'entretien et la maintenance de la pompe après son installation (ou sa réhabilitation), le village ou la population des usagers met en place, un système continu de mobilisation de ressources. Dans ce cadre, des cotisations sont systématisées et collectées de façon périodique dans certains villages. C'est le cas par exemple de Tissirougah, dans Ouassa-Péhunco, de Pikiré, dans Matéri, et de Kotopouga, dans Natititingou. La mobilisation des cotisations rencontre cependant, dans certaines localités, d'énormes difficultés. Jusqu'en 2000, dans la commune de Natitingou, des ménages entiers ont refusé de verser leur cotisation. Dans de nombreux villages cotonniers, ces cotisations sont mobilisées par les groupements villageois, sur les ristournes du coton-graine (cas de Ouoré, Brignamaro et Kérou-centre dans la commune de Kérou).

La vente de l'eau à la pompe aux usagers, constitue l'option de plus en plus utilisée dans plusieurs villages, pour assurer une continuité dans l'entretien et la maintenance des équipements de fourniture d'eau (cas de Kotopounga et Wètipounga dans Natitingou et de Bahoun dans Matéri). Cette option reçoit toutefois, avant son application, la validation de la population des usagers. Les modalités de sa mise en œuvre, en particulier la fixation du prix de l'eau, sont déterminées en concertation entre le comité de gestion et la population. En cas de vente de l'eau aux usagers, le comité de gestion, en concertation avec ceux-ci, met en place un système de gestion, qui a recours au service d'un gérant rémunéré. Les recettes issues de cette vente, sont épargnées dans le compte du comité de gestion. La rémunération du gérant du point d'eau, est directement supportée par ce compte. Dans le village de Kotopounga (Natitingou) par exemple, le comité de gestion a procédé à la désignation d'une personne âgée du village, non membre du comité local pour être gérant de la pompe. Ce dernier perçoit chez les usagers à la pompe, une redevance fixée à 10 FCFA pour 20 litres d'eau. Tous les soirs, il fait le point avec le trésorier et lui verse les recettes de la journée, en présence du secrétaire du comité. A la fin du mois, le trésorier verse les recettes totales de la période dans le compte du comité. Ces recettes s'élèvent à une moyenne de 300 FCFA par jour, soit 9000 FCFA par mois. La rémunération mensuelle de ce gérant varie en fonction de l'importance des recettes du mois. Cette rémunération se situe souvent dans une fourchette comprise entre $1000 \mathrm{~F}$ à 2500 FCFA.

49 Tout comme les AUE, les organisations d'usagers d'eau autour des comités de gestion de simples ouvrages, peuvent être assimilées à des entreprises sociales. En effet, l'initiative de mettre en place les équipements provient en général des usagers. Le service fourni à travers ces équipements reste ouvert à toute la communauté villageoise. Pour équilibrer leurs budgets, ces organisations combinent des ressources marchandes (recettes issues de la vente d'eau), des ressources non marchandes (subventions de l'Etat et d'autres partenaires, cotisations des ménages) et des ressources non monétaires (participation bénévole des membres du comité de gestion). Elles font usage de services rémunérés des gérants de point d'eau. Elles ont un mode de fonctionnement démocratique, notamment en matière de choix des dirigeants (membres du comité de gestion) et de prise de décisions (fixation du prix de l'eau). 
50 A travers la formation de ressources humaines qualifiées (responsables d'hygiène, responsables villageois de pompe et artisans réparateurs locaux), le dispositif mis en place autour des simples ouvrages de fourniture d'eau comporte des éléments favorables à la pérennisation du service dans les villages.

51 L'analyse des conditions de participation financière des populations dans la mise en place et l'entretien des équipements de fournitures d'eau potable dans certains villages de l'Atacora, met en relief le rôle particulièrement important des groupements villageois dans les localités de production cotonnière. C'est en effet par le biais de ces organisations économiques, que les fonds collectifs sont mobilisés pour le financement de nombre de réalisations communautaires dans ces localités. En matière de financement du développement local, cette situation privilégie les populations des villages cotonniers (départements du centre et du nord du pays), par rapport à celles des autres localités (cas du département de l'Atlantique) qui ne disposent pas encore de filière organisée, à l'image du coton.

52 L'analyse des formes de gouvernance de l'eau potable en zone rurale béninoise laisse apparaître, ces dernières années, un transfert de la gestion des infrastructures de fourniture de cette ressource, aux communautés organisées en associations d'usagers. Ces derniers assument, de façon commune, la propriété du système d'exploitation de l'eau dans leurs localités. Par le biais d'exploitants ou de gérants rémunérés, ils assurent la gestion au quotidien de la fourniture de la ressource. A travers des comités représentant les usagers, les communautés villageoises suivent et participent à cette gestion.

53 Dans les formes de gouvernance de la ressource, L'Etat et les collectivités locales assument, aux côtés des communautés, leurs missions d'acteurs publics dans le cadre d'un partenariat public/privé. Ainsi, par exemple, l'Etat conserve toujours dans le domaine sa responsabilité en matière de planification, de gestion de la ressource et de réglementation du secteur au plan national. Quant aux communes, les textes de lois sur la décentralisation prévoient leur réelle implication dans la mise en place des infrastructures hydrauliques sur leurs territoires. Dans les plans de développement que viennent d'élaborer ces communes, les priorités relatives à l'approvisionnement en eau potable des populations ont émergé dans beaucoup de cas.

54 L'analyse du fonctionnement concret des systèmes d'approvisionnement en eau potable dans les villages, fait apparaître un élément spécifique à la tradition rurale béninoise et un autre de nature importée. En effet, la propriété commune de certaines ressources naturelles, en particulier l'eau et la gestion communautaire de celles-ci, constituent des réalités propres aux zones rurales. Le respect de ce type de propriété et la coordination de cette gestion, sont en partie gouvernés par des normes coutumières. Quant à la pratique de vente de l'eau (valorisation par le prix), il s'agit d'une réalité importée en zone rurale. Traditionnellement, l'eau n'est pas un bien marchand dans les villages et ne peut être échangée contre de la monnaie. La mise en œuvre de cette forme de valorisation constitue dans plusieurs villages, une révolution à laquelle certains ménages ont, au départ, du mal à adhérer.

55 Par ailleurs, l'introduction de la dynamique élective, dans le système de gestion de l'eau, à travers le choix des membres de comités de gestion, constitue un mode de fonctionnement assez récent dans la gouvernance des ressources en zone rurale. Comparativement au mode traditionnellement répandu de choix par consensus, elle introduit des coût de transaction beaucoup plus élevés, du fait par exemple des rivalités 
et des négociations qui accompagnent des choix électifs dans certains villages. Enfin, les organisations mises en place par les usagers de l'eau (AUE, comité de gestion) présentent des caractéristiques assez proches des entreprises sociales définies par le réseau EMES. Elles peuvent donc être assimilées à des entreprises sociales de l'eau en milieu rural béninois.

\section{BIBLIOGRAPHIE}

Banque Mondiale (1994) : Rapport sur le développement dans le monde. Paris, Economica.

Benkler Y. (2003) : «The Political Economy of Commons», Upgrade, vol. 4, n³, june 2003, Open Knowledge, http://www.upgrade-cepis.org/issues/2003/3/upgrade-vIV-3.html.

Bertacchini Y., 2002, "Concertation territoriale et politique territoriale concertée », Laboratoire Le Pont, Département Services et Réseaux de Communication, Université de Toulon et du Var, 6 pages.

Berkes F., Feeny D., McCay B.J., Acheson J.M., 1989, « The benefits of the commons ", Nature, $\mathrm{n}^{\circ}$ 340, pp. 91-93.

Briand A. et Lemaitre A., 2004, « Privatisation de la distribution de l'eau potable en Afrique : une aubaine? » Actes de la journée d'études «Les territoires de l'eau », Université d'Artois, Arras, 26 mars 2004, pp. 98-115.

Bromley D.W., 1991, Environment and Economy. Property Rights and Public Policy, Cambridge- MA, Basil Blackwell.

Buchanan J., Musgrave R., 1999, Public finance and Public Choice: two Contrasting Visions of the State. Cambridge Mass., MIT Press, $272 \mathrm{p}$.

Calvo-Mendieta I., 2004, « Conflits d'usage dans la gestion des ressources en eau: analyse territoriale des modes de régulation », Actes de la journée d'études "Les territoires de l'eau », Université d'Artois, Arras, 26 mars 2004, 16 pages.

Calvo-Mendieta I., Dziedzicki J-M., Larrue C., 2002, « Case Study 1 : L'Audomarois basin », Contribution to European Water Regimes and the Notion of Sustainable Status (EUWARENESS) research project, final report, 47 pages.

Collignon B., Vezina M., 2000, « Les opérateurs indépendants de l'eau potable et de l'assainissement dans les villes africaines ", Synthèse d'une étude menée dans 10 pays d'Afrique, sous la conduite du Programme Eau et Assainissement, 13 p.

Cornes R., Sandler T., 1996, The Theory of Externalities, Public Goods and Club Goods, Cambridge University Press, $303 \mathrm{p}$.

Enterprise Works World Wide, 2003, «Eau potable », www.enterpriseworks.org, 2 p.

Feuillette S., 2004, « L'eau en France: entre subsidiarité et gestion spatiale ». Cybergeo, http:// www.cybergeo.presse.fr/eauville/Feuillette.htm. 3 pages.

Gauthier A-M., 2004, « De l'eau pour tous », Amis de la Mission, n4, 3 p. 
Hardin G., 1968, « The tragedy of the commons », Science, n¹62, pp. 1243-1248.

Hounmenou B., à paraître, Décentralisation, Gouvernance participative et dynamiques locales de développement économiques - Etude de cas en zones rurales au Bénin, Thèse de doctorat en Sciences Economiques, Université de Versailles Saint Quentin-en- Yvelines.

Hounmenou B., 2003, « Nouveaux modes de coordination des acteurs dans le développement local - Cas des zones rurales au Bénin », Développement Durable et Territoires, Dossier 2, http:// developpementdurable.revues.org, 25 pages.

Jaglin S., 2001, «L'eau potable dans les villes en développement : les modèles marchands face à la pauvreté », In : Les nouvelles politiques de l'eau : enjeux urbains, ruraux, régionaux, Revue Tiers Monde, $n^{\circ} 166$, avril- juin 2001.

Jaglin S., 2004, «Vingt ans de réformes dans les services d'eau urbains d'Afrique subsaharienne : une géographie de la diversité », Cybergeo, http://www.cybergeo.presse.fr/eauville/Jaglin.htm, 4 p.

Lych O.J., 1999, « Promoting legal recognition of community based property rights, including the commons: some theoretical considerations ", Paper presented at a Symposium of the International Association for the study of Common Property and the Workshop in Political Theory and Policy Analysis, Indiana University, Bloomington, Indiana, June 7, $4 \mathrm{p}$.

MATE, 2002, Les biens publics - Fiche1 : l'approche économique, Paris, Ministère de l'Aménagement du Territoire et de l'Environnement, Direction des études économiques et de l'évaluation environnementale, Février 2002, 6 p.

OCDE, 1999, Les entreprises sociales, Paris, Organisation de Coopération et de Développement Économiques. $70 \mathrm{p}$.

Olson M., 1965, The logic of collective action, public goods and theory of groups, Havard University Press, $186 \mathrm{p}$.

Ostrom E., 1990, Governing the commons. The Evolution of Institutions for Collective Action, Cambridge University Press, $280 \mathrm{p}$.

Ostrom E., 1999, « Coping with tragedies of the commons », American Review of Political Science, 2, pp. 493-535.

Stevenson G.G., 1991, Common Property Economics. A General Theory and Land Use Applications, Cambridge, Cambridge University Press, 256 pages.

Thaithe A., 2001, «L'eau : besoin, droit ou quel bien public? ", Colloque Les biens publics mondiaux, Association Française de Science Politique, Section d'Études Internationales, Pau, 25-26 octobre. 7 pages.

\section{NOTES}

1. Cette carte est extraite des travaux de thèse de Hounmenou (à paraître). Ces travaux ont été conduits entre 2002 et 2004, dans certaines communes des départements des Collines au centre et de l'Atlantique au sud (partie orange sur la carte).

2. Le Franc CFA est relié à l'Euro par la formule : 1 Euro = 655.9 FCFA.

3. EMES : Réseau européen d'émergence des Entreprises Sociales. 


\section{RÉSUMÉS}

Dans la fourniture des services de base aux populations, les politiques publiques de plusieurs pays, en particulier celles des Pays en Voie de Développement (PED), ont connu ces dernières années une profonde mutation. Les changements les plus remarquables se rapportent à la remise en cause du service public gratuit. Outre la nécessité fondamentale pour les Etats d'assainir leurs économies, ces réformes visent à favoriser une participation des populations dans la conduite des opérations ayant pour objectif la satisfaction de leurs besoins. La question de la participation constitue actuellement un élément capital pour l'organisation des services au niveau communautaire. En effet, plusieurs expériences ont montré que les projets réalisés sans la participation des populations concernées ont échoué au moment de l'exécution ou, faute d'entretien, n'ont eu que des retombées éphémères (Banque Mondiale, 1994). Au Bénin, certaines opérations de développement, en particulier celles conduites dans le secteur de l'approvisionnement en eau potable des populations rurales, n'ont pas échappé à cette réalité. En effet, jusqu'à la fin des années 1980, plusieurs ouvrages d'approvisionnement en eau potable ont été construits par les pouvoirs publics, sans une réelle participation des communautés bénéficiaires en milieu rural béninois. Cette situation a occasionné un manque d'intérêt des populations, qui s'est exprimé par l'abandon des ouvrages en cas de panne, et le recours à l'utilisation de sources d'eau non potable. Pour y remédier, le Bénin a opté en 1992 pour une nouvelle stratégie nationale d'alimentation en eau potable. La stratégie a pour objectif l'implication des populations du monde rural dans tout le processus d'appropriation de l'alimentation en eau. Les principes fondamentaux de cette stratégie sont notamment, la décentralisation du processus de prise de décision ainsi que la participation des communautés à l'investissement et à la gestion des points d'eau. La concrétisation de cette volonté d'améliorer les systèmes d'alimentation en eau potable se traduit par exemple, par l'adhésion des communautés à travers leurs contributions financières à la réalisation des ouvrages et la mise en place de structures de gestion chargées de l'entretien et de la maintenance de ces derniers. Les structures de gestion ont entre autres tâches, de mobiliser la participation financière des populations à la construction des ouvrages, de fixer le prix de l'eau, de choisir les vendeurs et de gérer la maintenance des équipements. La nouvelle stratégie se traduit aussi par l'entrée dans le système local lié à la gouvernance de l'eau potable en zone rurale, de nouveaux acteurs tels que les collectivités locales (les nouvelles communes), les ONG, les entreprises du secteur privé et les organisations d'usagers d'eau, qui ont un fonctionnement assez proche de celui des entreprises sociales. A partir d'éléments empiriques, cet article se focalise sur les analyses liées aux dynamiques socio-économiques des acteurs impliqués dans le processus d'approvisionnement en eau des populations rurales au Bénin.

In populations' basic service supplying, the public policies, especially those of developing countries, have met deep changes in the last recent years. The most important changes are relative to the withdrawal of gratuitous public service. Beside the fundamental necessity of states to clean their economies, those reforms aim to favour populations' participation in the management of operations linked to their needs satisfaction. The participation question constitutes actually a major element, for services organisation at community level. Indeed, several experiences have shown that the projects realised without the concerned populations' 
participation, have failed at the execution moment, or by the lack of maintenance, they have led to negative results (Banque Mondiale, 1994). In Benin, certain development operations, especially those conducted in the drinking water supply sector, don't escape to that reality. Indeed, until the end of the 1980's, many drinking water supply equipment have been constructed by public authorities, without a real participation of rural area beneficiary communities. This situation has led to a lack of populations' interest, expressed by the withdrawal of equipment in case of breakdown, and the turning to the use of non drinking water source. In order to remedy this situation, Benin Republic has opted in 1992 for a new national strategy of drinking water supply. This strategy aims the implication of rural area populations in the process of water supplying. The major principles of the strategy are among other things, the decentralisation of decision making process, as well as communities' participation to investment and water point management. The materialisation of this willingness to improve the drinking water supply systems is expressed for example, by the communities joining through their contributions to equipment realisation and equipment management structures' installation. The management structures' tasks are among other things, the mobilisation of populations' financial participation to equipment' construction, the water price determination, the water sellers' choice and the equipment' management. The new strategy is also materialised by the arrival into the local system of drinking water governance in rural area, of new actors such as, local authorities, NGO, and water users organisations which have a functioning of "social enterprises". From empirical elements, this paper is focussed on the analysis linked to the socio-economic dynamic of actors implied in Benin rural populations' water supply process.

\section{INDEX}

Mots-clés : approvisionnement en eau, structures de gestion, association d'usagers d'eau, gouvernance de l'eau, dynamiques locales, propriété commune, entreprises sociales

Keywords : water supply, management structures, water users association, water governance, local dynamics, common property, social enterprise

\section{AUTEUR}

\section{BERNARD G. HOUNMENOU}

Bernard G. Hounmenou est ingénieur agro-économiste, titulaire d'un DEA en développement soutenable intégré (option, développement économique et social) de l'Université de Versailles Saint-Quentin-en-Yvelines, il termine actuellement une thèse de doctorat en sciences économiques au Centre d'Economie et d'Ethique pour l'Environnement et le Développement (C3ED, UMR 063 IRD-UVSQ). Ses recherches portent sur le thème « Décentralisation, gouvernance participative et dynamiques locales de développement- Etude de cas en milieu rural au Bénin ». C3ED, Université de Versailles Saint-Quentin-en-Yvelines, 47, Bd VAUBAN- 78047 GUYANCOURT CEDEX - France. hbenaf@yahoo.fr 